\title{
Laboratory Analogues of the Carbonaceous Dust: Synthesis of Soot-like Materials and their Properties
}

\author{
T. Pino ${ }^{1}$, Y. Carpentier ${ }^{1} \dagger$, G. Féraud ${ }^{1}$, Ph. Bréchignac ${ }^{1}$, \\ R. Brunetto ${ }^{2}$, L. d'Hendecourt ${ }^{2}$, E. Dartois ${ }^{2}$ and J.-N. Rouzaud ${ }^{3}$ \\ ${ }^{1}$ Institut des Sciences Moléculaires d'Orsay, CNRS - Univ Paris-Sud, F91405 Orsay Cedex, \\ France \\ email: thomas.pino@u-psud.fr \\ ${ }^{2}$ Institut d'Astrophysique Spatiale, CNRS - Univ Paris-Sud, F91405 Orsay Cedex, France \\ ${ }^{3}$ Laboratoire de Géologie, Ecole Normale Supérieure, F75231 Paris Cedex 5, France
}

\begin{abstract}
Carbonaceous cosmic dust is observed through infrared spectroscopy either in absorption or in emission and the details of the spectral features are believed to shed some light on its structure and finally enable the study of its life cycle. Other spectral domains also contain some information, as does the UV bump at $217 \mathrm{~nm}$. In order to progress on the understanding of these spectral features, many laboratory works are devoted to the production and characterization of laboratory analogues. Generally several analytical tools are used in combination to better analyse the intimate structure of the analogues and the influence of the nanostructuration on the spectral properties. In this proceeding We will focus on the elaboration of new spectral parameters that enables the nanostructuration of the carriers of the AIBs to be traced.
\end{abstract}

Keywords. ISM: general, astrochemistry, (ISM:) dust, extinction, infrared: ISM

\section{Introduction}

Carbonaceous materials are observed in many objects in space (Henning \& Salama 1998, Ehrenfreund \& Charnley 2000) through their specific spectral features. Some are found in the visible to UV range (Désert et al. 1990, Draine 2003, Zubko et al. 2004), but most of the spectral information is obtained in the infrared range of wavelength. In particular, the aromatic infrared bands (AIBs) (Gillett et al. 1973) trace carbonaceous particles whose inferred size vary from large molecules, the polycyclic aromatic hydrocarbons (PAHs), to nanoparticles of a few nanometers (Léger \& Puget 1984, Crawford et al. 1985, Tielens 2008). Three main classes A, B and C of astrophysical spectra have been proposed based on the observed spectral characteristics in the 6 to $9 \mu \mathrm{m}$ wavelength region (Peeters et al. 2002). If the band positions, in most sources, are clearly attributed to aromatic materials (Goto et al. 2007), however, the exact nature of the emitters still escape. In the case of the class A emitters, the carbonaceous species emit through their vibrational bands thanks to the stochastic heating mechanism (Puget \& Léger 1989, Allamandola et al. 1989, Draine \& Li 2001). In the other cases, thermal emission may occur, particularly for the class $\mathrm{C}$ objects. In order to study these materials, many laboratory astrophysics works are perfomed on various analogues. At Orsay (France), we focus on the polyaromatic carbons with a special emphasize on the soot-like nanoparticles. Such materials provide interesting laboratory analogues of cosmic dust. The soot samples are

$\dagger$ Present address: Laboratoire de Physique des Laser, Atomes et Molécules, Bât P5 - USTL, F-59655 Villeneuve d'Ascq cedex, France 
produced using a low-pressure flame, flat and premixed. The nanostructural characterisation and their spectral signatures through the positions of infrared bands are described and shown to provide a spectral index of the shape of the polyaromatic units. The astrophysical implications of such index are thus discussed. The detailed analysis is reported elsewhere (Carpentier et al. 2012).

\section{A spectral index for the shape of the polyaromatic units}

Only spectroscopic parameters extracted from the infrared bands can be used to decipher the intimate structure of the carriers of the AIBs. Using our soot, we explore the details of the variations of the infrared bands combining Raman spectroscopy and HRTEM images (not shown here) in order to link the spectral features to specific structural properties of the polyaromatic units within the soot. We have recently shown that the $6.2 \mu \mathrm{m}$ band position traces the intimate cross-linkage of the polyaromatic units composing the soot (Pino et al. 2008). Such evolution is accompanied by an evolution of the band at about $8 \mu \mathrm{m}$. The correlation between the 6.2 and $8 \mu \mathrm{m}$ band is shown in Fig. 10 in Carpentier et al. 2012. We tentatively attribute this band to defect-like modes of the polyaromatic units. The defects are located either at the edge and cross-linking the units together, or within the unit and shaping the unit away from planarity. Such nanostructural characteristics are, in combustion processes, related to the growth mechanism of the soot (D'Anna 2009). Similarly, different interstellar emitters could have experienced different formation pathways.

In most analysis, the spectral parameters are used to build an aromatization index when dealing with polyaromatic materials. It is necessary because most of the properties are determined by the size of the polyaromatic units and the $s p^{2}$ to $s p^{3}$ carbon ratio, and the aromatic index increases with both. However, in the present soot samples, size

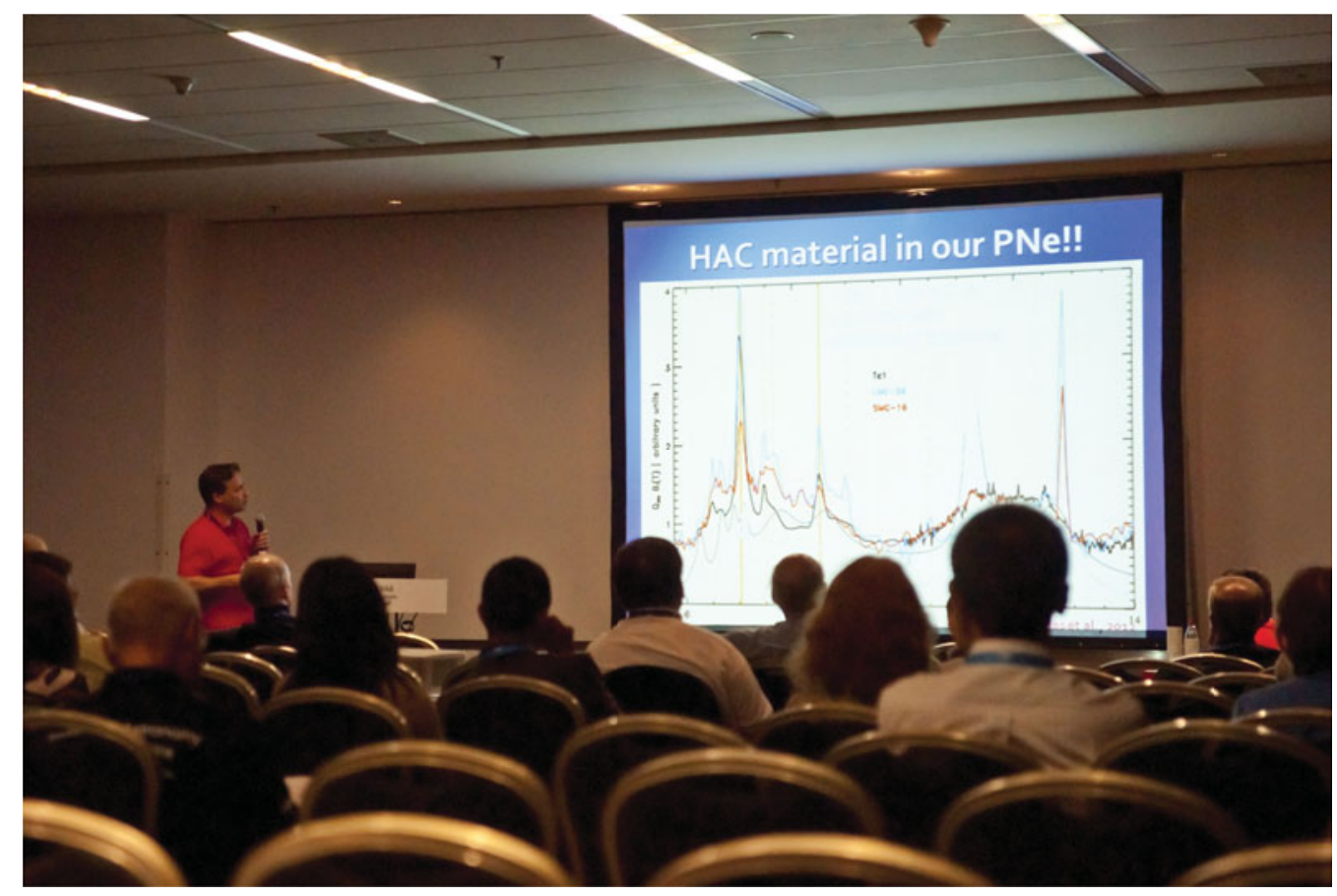

Figure 1. Jan Cami giving a talk on interstellar fullerenes. 
variation is not important. In addition, the mean size is always below $2 \mathrm{~nm}$ and precludes the use of any reliable index, whatever the experimental methods. For such polyaromatic samples, it is more important to consider the shapes of the polyaromatic units which are found from the study of their nanostructuration. It appears that the $8 \mu \mathrm{m}$ band position is a good tracer of the defects that build curvature and tortuosity for the polyaromatic units. The correlation of its position with that of the $6.2 \mu \mathrm{m}$ band shows that the latter band position is also strongly influenced by these defects. Thus the positions of the two bands can be seen as a (nano)structural index.

\section{Conclusions}

Thanks to soot analogues of the cosmic dust, the meaning of the spectral characteristics of these AIBs, classified from A to C, can be deeply studied in the laboratory. The role of the defects in the carbonaceous particles appears to be at the core of the variations of the spectral features, in particular those related to the aromatic carbon skeleton: the band at about $6.2 \mu \mathrm{m}$ and the band at about $8 \mu \mathrm{m}$. Their positions are sensitive to the (nano)structuration. Progress on the understanding of the nanostructuration of the emitters will be essential to decipher the growth and lifecycle of this component of the interstellar medium, as proposed in Carpentier et al. 2012.

\section{Acknowledgements}

This work was supported by the French national program "Physique et Chimie du Milieu Interstellaire" and the French "Agence Nationale de la Recherche" (contract ANR10-BLAN-0502).

\section{References}

Allamandola, L. J., Tielens, A. G. G. M., \& Barker, J. R. 1989, ApJS 71, 733

Carpentier, Y., Féraud, G., Dartois, E., Brunetto, R., Charon, E., Cao, A.-T., d'Hendecourt, L., Bréchignac, P., Rouzaud, J. N., \& Pino, T. 2012, A\& A 548, 40

Crawford, M., Tielens, A., \& Allamandola, L. 1985, ApJ 293, L45

D'Anna, A. 2009, in: H. Bockhorn, A. D'Anna, A. F. Sarofim \& H. Wang (eds.), Combustion Generated Fine Carbonaceous Particles, Proc. of an International Workshop (Villa Orlandi, Anacapri: KIT Scientific Publishing), p. 289

Désert, F.-X., Boulanger, F., \& Puget, J. L. 1990, A\&A 237, 215

Draine, B. T. 2003, ARAA 41, 241

Draine, B. T. \& Li, A. 2001, ApJ 551, 807

Ehrenfreund, P. \& Charnley, S. B. 2000, ARAA 38, 427

Gillett, F. C., Forrest, W. J., \& Merrill, K. M. 1973, ApJ 183, 87

Goto, M., Kwok, S., Takami, H., Hayashi, M., Gaessler, W., Hayano, Y., Iye, M., Kamata, Y., Kanzawa, T., Kobayashi, N., Minowa, Y., Nedachi, K., Oya, S., Pyo, T.-S., Saint-Jacques, D., Takato, N., Terada, H., \& Henning, T. 2007, ApJ 662, 389

Henning, T. \& Salama, F. 1998, Science 282, 2204

Léger, A. \& Puget, J. 1984, A\& A 137, L5

Peeters, E., Hony, S., van Kerckhoven, C., Tielens, A. G. G. M., Allamandola, L. J., Hudgins, D. M., \& Bauschlicher, C. W. 2002, A\&A 390, 1089

Pino, T., Dartois, E., Cao, A.-T., Carpentier, Y., Chamaillé, T., Vasquez, R., Jones, A. P., d'Hendecourt, L., \& Bréchignac, P. 2008, A\&\& 490, 665

Puget, J. L. \& Léger, A. 1989, ARAA 27, 161

Tielens, A. G. G. M. 2008, ARAA 46, 289

Zubko, V., Dwek, E., \& Arendt, R. G. 2004, ApJS 152, 211 